\title{
MODELLING OF TEXTURE DEVELOPMENT AND DEFORMATION MECHANISMS IN A TI20V ALLOY USING A SELF CONSISTENT POLYCRYSTAL APPROACH
}

\author{
S. MERCIER, L. S. TÓTH and A. MOLINARI \\ Laboratoire de Physique et Mécanique des Matériaux URA CNRS $N^{\circ} 1215$, \\ ISGMP-Université de Metz, Ile du Saulcy, 57045 Metz, France
}

(Received 5 June 1995; in final form 20 July 1995)

\begin{abstract}
Modellisations of the deformation texture development in a Ti20V alloy (20 volumic \% Vanadium) during rolling have been carried out using the viscoplastic self consistent model of Molinari and Tóth (1994). The model parameters and the slip and twinning systems were identified from experimental data. The experimental texture evolution is well reproduced with only a small contribution of the twinning activity.
\end{abstract}

KEY WORDS: Titanium, cold rolling, self consistent modelling, twinning, work hardening.

\section{INTRODUCTION}

There are three approaches available for the prediction of the plastic behaviour of a polycrystalline medium: the Taylor model, the self consistent approach and finally the finite element method combined with crystal plasticity. When average macroscopic properties are sought for, the Taylor model gives an upper bound and becomes unrealistic when large strains are involved. Precise results can be obtained by the finite element technique but the complexity of the calculations in conjunction with crystal plasticity renders them still difficult to handle, especially in 3D simulations. Nevertheless, such efforts have already been made (Mathur et al. (1989)). For the moment, it is the self consistent scheme which can be used the most efficiently.

This latter model has been developed on the basis of the Eshelby solution derived in linear elasticity (Eshelby (1957)) which describes the behaviour of an inclusion embedded into an infinite matrix. The Eshelby solution is also extended to the case of polycrystal plasticity using a homogenisation scheme. In this approach, the grain interactions with the matrix are taken into account through an interaction law. Each grain is considered as an elliptical inclusion which interacts with the matrix. The latter is also called as the homogeneous equivalent medium (HEM) having the average properties of the polycrystalline sample. In this way, it is possible to calculate the behaviour of a grain within the matrix. The next step is to derive the macroscopic behaviour of the whole medium for which purpose an averaging of the different 
properties of each grain can be carried out. Furthermore, it has to be assured that the average deformation gradient made over all constituent grains of the polycrystal is equal to the deformation gradient imposed on the sample. In the present paper the self consistent approach proposed by Molinari and Tóth is employed (1994). This model is an improved version of the approach developed by Molinari et al. (1987) with an important difference that it is tuned to some results obtained by the finite element technique.

The material considered in the present work is a Ti-20\%V alloy. This material has a b.c.c. crystallographic structure at room temperature with a significant activity of the $\{332\}$ twinning family. The aim of the present study is to examine the role of the different slip and twinning systems on the evolution of the deformation texture during cold rolling.

\section{THE SELF CONSISTENT MODEL}

Molinari and Tóth have proposed the following interaction law between a grain and the matrix (1994):

$$
\mathbf{S}^{\mathbf{g}}-\mathbf{S}=\alpha\left[\Gamma^{\mathrm{sgg}-1}+\mathbf{A}^{\mathbf{s}}\right]\left[\mathbf{d}^{\mathbf{g}}-\mathbf{D}\right]
$$

Here $d^{\mathrm{g}}$ and $s^{\mathrm{g}}$ are the strain rate and the deviatoric stress in the grain respectively, $\mathbf{D}$ and $\mathbf{S}$ are the same quantities for the matrix. $\mathbf{A}^{\mathbf{s}}$ is the secant modulus defined by $\mathbf{S}=\mathbf{A}^{\mathrm{s}}: \mathbf{D}$, while the tensor $\Gamma^{\mathrm{sgg}^{-1}}$ accounts for the shape of the grain during deformation. The parameter $\alpha$ has been introduced by Molinari and Tóth in order to tune the self consistent model to the finite element method. This has been done for the case of a spherical grain embedded into an infinite matrix. With a special choice of $\alpha$, the new constitutive model can reproduce all previous approaches:

\begin{tabular}{ccccc}
\hline$\alpha:$ & 0 & $m$ & 1 & $\infty$ \\
\hline model: & static & tangent & secant & Taylor \\
\hline
\end{tabular}

The finite element based $\alpha$ parameter lies in the range of 0 and 1 and depends in a first approximation on the value of the strain rate sensitivity index $\mathrm{m}$ (Molinari and Tóth (1994)). $\alpha$ may also depend on the strain path, however, such a possible dependence has not yet been explored, so the results of the analysis carried out for tensile defomation is employed in the present work:

$$
\alpha=1-0.4(m-1)^{2} .
$$

Hardening is also accounted for in the present modelling using microscopic slip system hardening laws. Self and latent hardening of the slip systems is considered in the following way (Bronkhorst et al. (1992)):

$$
\dot{\tau}_{0}^{\mathrm{i}}=\sum_{\mathrm{j}} H^{\mathrm{ij}}\left|\dot{\gamma}^{\mathrm{j}}\right| \quad i, j=1 . . n
$$


where

$$
\mathrm{H}^{\mathrm{ij}}=\mathrm{q}^{\mathrm{ij}} \mathrm{h}_{0}\left(1-\frac{\tau_{0}^{\mathrm{i}}}{\tau_{\text {sat }}}\right)^{\mathrm{a}},
$$

and $n$ is the total number of slip and twinning systems; $q^{i j}$ is the hardening matrix. It describes the influence of a system $i$ on the plastic activity of a system $j$; it is defined as follows:

$\mathrm{q}^{\mathrm{ij}}=1$ for coplanar systems,

$\mathrm{q}^{\mathrm{ij}}=1$ for systems with parallel glides,

$\mathrm{q}^{\mathrm{ij}}=1.4$ for systems with perpendicular gliding directions,

$\mathrm{q}^{\mathrm{ij}}=1.2$ for all other cases.

This type of construction of the hardening matrix has been proposed recently by Zhou et al. (1993). $\tau_{0}^{\mathrm{i}}$ in eqn (4) is the reference stress in the rate sensitive constitutive law for rate dependent crystallographic slip:

$$
\dot{\gamma}^{\mathrm{i}}=\dot{\gamma}_{0} \operatorname{sgn}\left(\tau^{\mathrm{i}}\right)\left|\frac{\tau^{\mathrm{i}}}{\tau_{o}^{\mathrm{i}}}\right|^{1 / \mathrm{m}} .
$$

$\tau^{i}$ is the resolved shear stress of the system $i$ and $\dot{\gamma}_{0}$ is a constant reference slip rate. The other parameters, $h_{0}, \tau_{s a t}$, and a can be obtained by matching the experimental stress-strain curves.

\section{MATERIAL PARAMETERS}

The self consistent scheme requires several physical factors to be known. These are:

- strain rate sensitivity index, $\mathrm{m}$,

- interaction parameter, $\alpha$,

- crystallographic texture (orientation distribution of the grains),

- operating slip and twinning systems,

- microscopic hardening parameters: $\dot{\gamma}_{0}, a, h_{0}, \tau_{\text {sat }}$.

These information were obtained by analysing the stress-strain curves measured in tension and compression (Guibert (1994)), from the measured textures as well as from experimental observations of the microstructure (Nicotra and Philippe (1994a, b)).

\subsection{Strain Rate Sensitivity}

The strain rate sensitivity exponent $m$ was deduced from the hardening curves obtained in tension at different strain rates. At a given strain and for two strain rates $\dot{\varepsilon}_{1}$ and $\dot{\varepsilon}_{2}$, the ratio of the corresponding stresses is equal to 


$$
\frac{\sigma_{1}\left(\dot{\varepsilon}_{1}\right)}{\sigma_{2}\left(\dot{\varepsilon}_{2}\right)}=\left(\frac{\dot{\varepsilon}_{1}}{\dot{\varepsilon}_{2}}\right)^{m},
$$

from which:

$$
m=\frac{\ln \left(\sigma_{1}\right)-\ln \left(\sigma_{2}\right)}{\ln \left(\dot{\varepsilon}_{1}\right)-\ln \left(\dot{\varepsilon}_{2}\right)}
$$

The experimental values give $m=0,01$. According to eqn. (2), for such an $m$ value, the interaction parameter $\alpha$ is equal to 0.6.

\subsection{Slip and Twinning Systems}

The Ti20V material is a two-phase alloy, consisting of the $\beta$ and the $\omega$ phase. As the latter phase is present only in a small volumic fraction, its effect on the mechanical properties and on the texture development is neglected in the present work. The $\beta$ phase has a b.c.c crystallographic structure and can accommodate the deformation by two different mechanisms: glide and twinning. The available slip systems in the Ti20V material are identified as $\{11 \overline{2}\}<111>$ and $\{110\}<\overline{1} 11>$ (well known families for b.c.c materials). The twinning systems have been identified by Nicotra and Philippe (1994a, b) and were found to be the family of $\{33 \overline{2}\}<113>$ (Hanada et al. 1985)). A complete list of these twinning systems is given in Table 1. It is important to note that two different critical shear strains are possible for this twinning family; $\gamma_{c}=0,714$ or $\gamma_{c}=3,5178$ (Richman, 1964).

\subsection{Hardening Parameters}

As stated above, two kinds of slip systems and one twinning family are available to produce plastic deformation in the present material. An important information, however, cannot be provided by microscopic observations, that is the value of the initial reference stresses of the slip and twinning systems. Moreover, the microscopic hardening parameters have also to be specified (see eqns (3) and (4)): $\dot{\gamma}_{0}, a, h_{o}, \tau_{\text {sat }}$. These

\begin{tabular}{|c|c|}
\hline Habit plane & Twinning direction \\
\hline $\begin{array}{ccc}\frac{3}{3} & 3 & \overline{2} \\
\frac{3}{3} & \frac{3}{3} & \frac{2}{2} \\
3 & \frac{3}{3} & 2 \\
\frac{3}{3} & \frac{2}{2} & \frac{3}{3} \\
\frac{3}{3} & 2 & \frac{3}{3} \\
\frac{3}{2} & 2 & \frac{3}{3} \\
2 & \frac{3}{3} & \frac{3}{3} \\
\frac{2}{2} & \frac{3}{3} & \frac{3}{3}\end{array}$ & $\begin{array}{lll}\frac{1}{1} & 1 & \frac{3}{3} \\
\frac{1}{1} & \frac{1}{1} & \frac{3}{3} \\
1 & \frac{1}{3} & \frac{3}{1} \\
\frac{1}{1} & 3 & \frac{1}{3} \\
\frac{1}{1} & \frac{3}{3} & \frac{1}{1} \\
1 & \frac{3}{3} & \frac{1}{1} \\
\frac{3}{3} & 1 & \frac{1}{3} \\
\frac{3}{3} & \frac{1}{1} & \frac{1}{3} \\
\frac{1}{1} & \frac{1}{1}\end{array}$ \\
\hline
\end{tabular}

Table 1 List of the $\{33 \overline{2}\}<113>$ twinning systems 


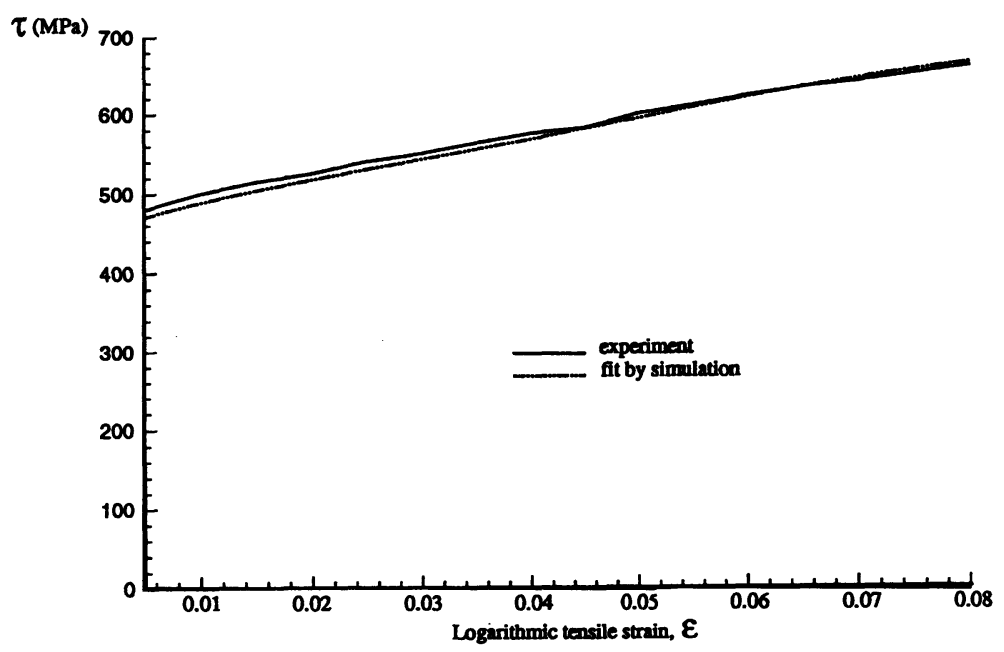

Figure 1 Comparison between the experimental and simulated tensile hardening curves $\left(\dot{\varepsilon}=\mathbf{0 . 0 2} \mathbf{s}^{\mathbf{1}}\right)$.

parameters as well as the reference stresses $\tau_{0}^{i}$ were determined with several simulations and comparisons with the experimental stress-strain curves. The tensile strain hardening curves obtained by Guibert (1994) were used for this proceduce (Figure 1). The initial critical stresses were chosen so that the yield stress of the material at zero strain is quite well reproduced. The critical stress for twinning was taken to be larger than the ones for slip in order to maintain the twinning activity smaller than slip. By varying the $\dot{\gamma}_{0}, a, h_{0}$ and $\tau_{\text {sat }}$ parameters in a series of iterative simulations, they were finally accepted when the experimental and the predicted hardening curves practically coincided (see Figure 1). The following set of parameters have led to the reproduction of the tensile hardening curve by the present polycrystal model:

$\begin{array}{lll}\tau_{0}^{110} & = & 192 \mathrm{MPa} \\ \tau_{0}^{112} & = & 187 \mathrm{MPa} \\ \tau_{0}^{332} & = & 527 \mathrm{MPa} \\ \tau_{\text {sat }} & =1300 \mathrm{MPa} \\ \dot{\gamma}_{0} & =1.0 \mathrm{~s}^{-1} \\ h_{0} & =600 \mathrm{MPa} \\ a & =3.1\end{array}$

It is evident that by properly adjusting these parameters, practically any polycrystal model would be able to reproduce the experimental hardening curve. The situation is not the same, however, for the texture development. As it will be shown in Section 4 below, the latter is very sensitive to the assumed model. 


\subsection{Anisotropy}

The texture in the sheets before deformation were measured using X-ray diffraction by means of pole figures $(\{110\},\{100\},\{112\})$. The initial Orientation Distribution Function (ODF) has been calculated from these pole figures by the ODF software of Van Houtte. It has to be noted that the presence of the $\omega$ phase, which is hexagonal, created some difficulties in the processing of the texture. This is because the reflections $\{11 \overline{2} 0\},\{02 \overline{2} 1\}$ and $\{03 \overline{3} 0\}$ coincide with the $\{110\},\{100\}$ and $\{112\}$ reflections, respectively, of the $\beta$ (bcc) phase. Nevertheless, as the volumic fraction of the $\omega$ phase is estimated not to be more than $10 \%$; the complete pole figures could well reproduce the measured incomplete ones. Concerning the ODFs, however, some minor components appeared (see Figures 2 and 3) which are due to the $\omega$ phase.

As can be seen from Figure 2, the initial distribution is far from random. The ODF reveals the existence of some preferential orientations in the undeformed material. It is therefore necessary to integrate this anisotropy into the simulations. For this purpose, the initial continuous ODF was discretised to 400 orientations employing the "statistical technique" of Tóth and Van Houtte (1992). This discretised texture was then used in all the model simulations as the initial texture.

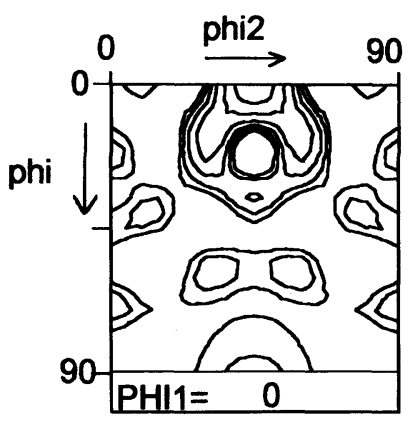

RD

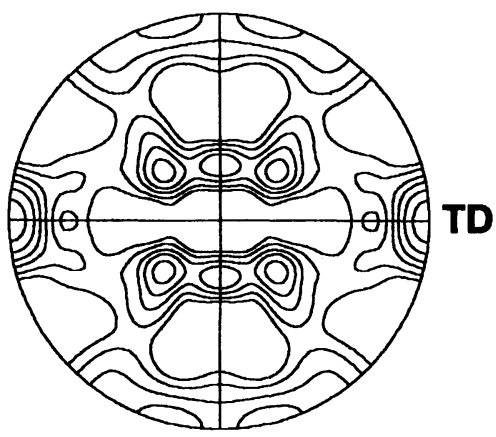

$\{110\}$

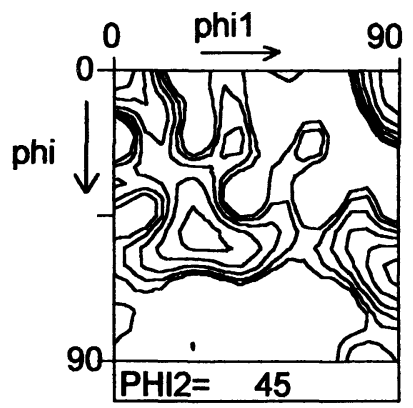

RD

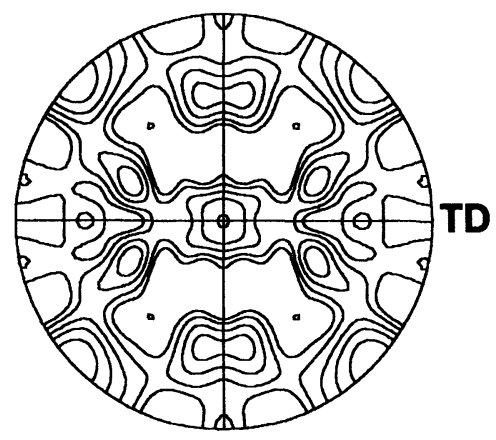

$\{100\}$

Figure 2 Two ODF sections and the two pole figures showing the initial texture in the sheet. Isovalues in the ODF: $1.0,1.4,2.0,2.8,4.0,5.6$. Isovalues in the pole figures: $0.8,1.0,1.3,1.6,2.0,2.5$. 

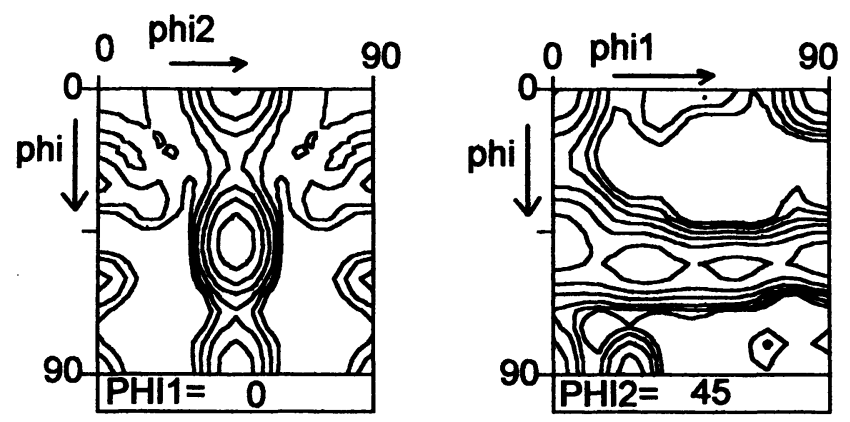

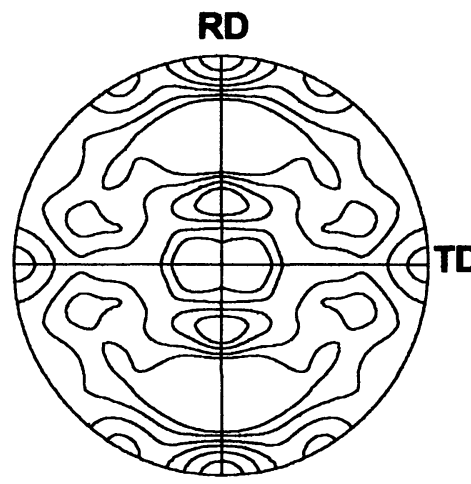

$\{110\}$

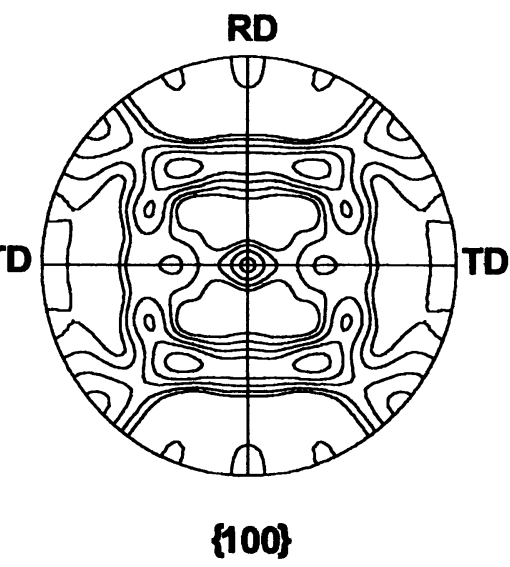

Figure 3 Two ODF sections and two pole figures showing the texture in the sheet after $50 \%$ thickness reduction. Isovalues in the ODF: $0.7,1.0,1.4,2.0,2.8,4.0$. Isovalues in the pole figures: $0.8,1.0$, $1.3,1.6,2.0,2.5$.

\section{APPLICATION TO ROLLING TEXTURES}

In the simulation procedure, hardening and the intial experimental grain orientation distribution were taken into account. The laboratory reference system was defined so that directions 1 and 2 coincided with the rolling and transverse directions, respectively. Large plastic deformation was achieved in small steps. The prescribed deformation gradient for the deformation of the sample was taken as:

$$
\left(\begin{array}{llc}
\dot{\varepsilon}_{11} & 0 & 0 \\
0 & 0 & 0 \\
0 & 0 & -\dot{\varepsilon}_{11}
\end{array}\right) \text {. }
$$

In a Taylor type of calculation, this deformation gradient is uniform, that is applied on the constituent grains of the polycrystal one by one. In the self consistent approach, this strain rate is only prescribed on the sample, that is, individual grains can deform differently. The deformation of a grain is governed by the interaction equation (eqn. (1)), but the average strain rate made over all grains is assured to reproduce the imposed 
strain rate given by eqn. 8 . The $12\{11 \overline{2}\}<111>$; $12\{110\}<\overline{1} 11>$ bcc slip systems and the $12\{33 \overline{2}\}<113>$ twinning systems were implemented into the numerical code. The twinning systems were also considered to be activated in the same way as the slip systems. However, any activity of twinning produces a part of the crystal to be reoriented into its twin-position. This phenomenon was also simulated in the present work using the Monte Carlo type approach first developed by Van Houtte (1978). In the present work, a grain was completely replaced by its twin orientation if the accumulated shear in a twinning system was large enough as compared to the required shear strain of the twinning procedure. The occurence of twinning was taken proportional to the accumulated shear in a given twinning system with respect to the critical shear. Twinning was produced as soon as a random number generator decided about the possibility of twinning. For large number of grain orientations, this type of Monte Carlo technique is equivalent to the so called "volume transfer scheme" developed by Tomé et al. (1991).

In the following, the predicted textures are displayed in form of ODFs as well as in pole figures and compared to the experimental rolling textures. The simulations were conducted at small strain rate (static conditions, as in the experiments) and up to an equivalent logarithmic thickness strain of 0,8 . This corresponds to $50 \%$ thickness reduction of the sheet. Before showing the results of the simulations, the experimental texture after rolling is described.

\subsection{Characteristics of the Texture After Rolling}

The measured texture after $50 \%$ thickness reduction is displayed in Figure 3. It has the classical features of a bcc rolling texture (Tóth et al. (1990)) (see Figure 4). Two
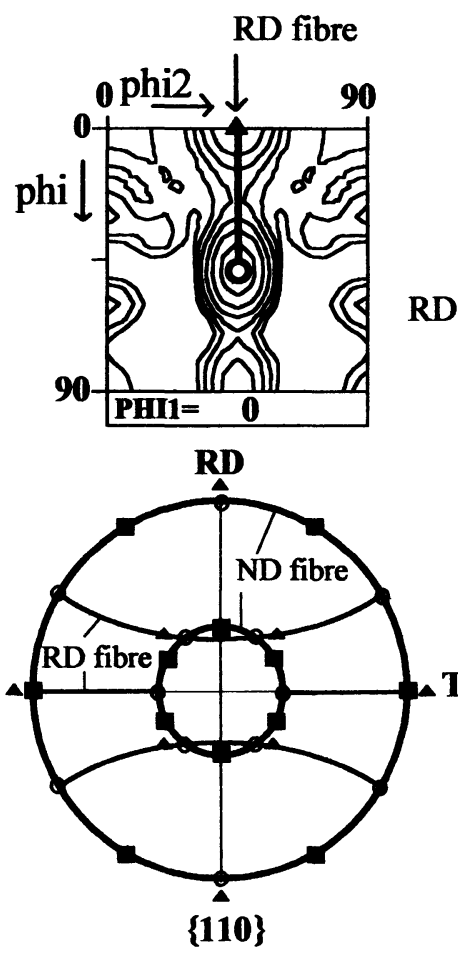
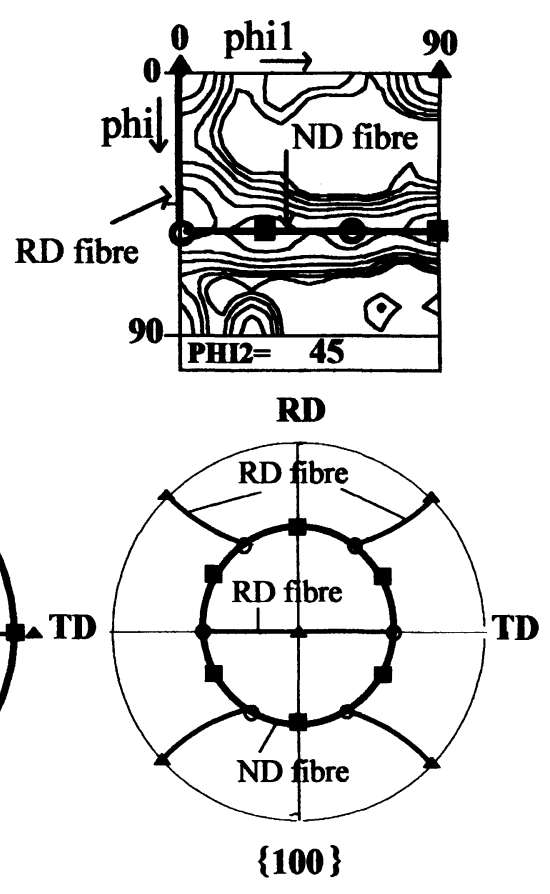

Figure 4 Key figures for the location of the ideal orientations of the b.c.c. rolling textures. Legend: $\bigcirc(111)[1 \overline{1} 0], \mathbf{1}(111)[1 \overline{2} 1], \Delta(001)[1 \overline{1} 0]$. 
stable and pronounced orientations, the $(001)<1 \overline{1} 0>$ and the $(111)<1 \overline{1} 0>$ appear in the $\varphi_{1}=0^{\circ}$ section of the ODF. Another feature of the texture is the existence of two fibres, well visible in the $\varphi_{2}=45^{\circ}$ section: one with $<1 \overline{10}>$ parallel to the rolling direction ('RD' fibre) and another one with the $<111>$ directions aligned parallel to the normal direction of the sample ('ND' fibre). The presence of the $\omega$ phase produces some minor "ghost" components in the ODF which are evident in the $\phi_{1}=0$ and $\phi_{2}=45^{\circ}$ sections in Figure 3. These are located near to the $\phi=90^{\circ}$ plane where there are usually no ideal orientations of the deformation texture in rolling of bcc metals. Therefore, these minor "ghost" components will be neglected in the discussion of the simulation results.

\subsection{Textures Obtained by the Self Consistent Model}

Using the microscopic hardening parameters derived in section 3.3 above together with the initial grain orientation distribution (400 grains), the development of the deformation texture has been simulated up to a thickness reduction of $50 \%$. The obtained texture is shown in Figure 5. By comparison with the experimental texture (Figure 3) it is

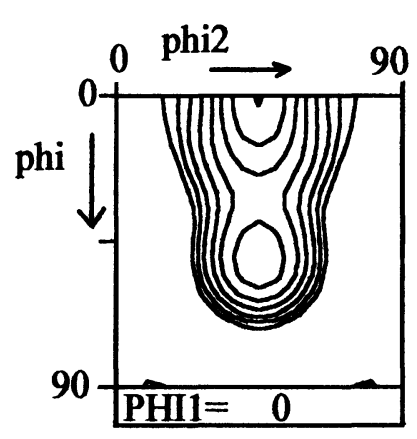

RD

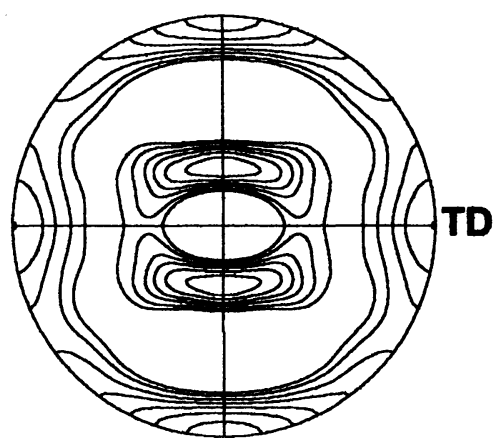

$\{110\}$

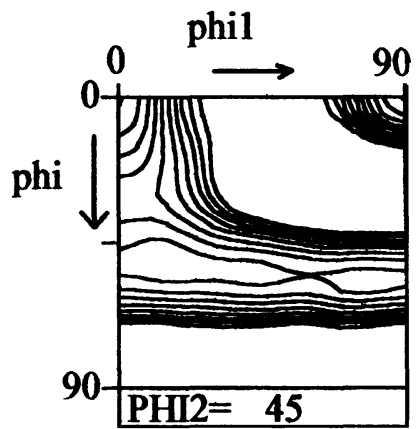

RD

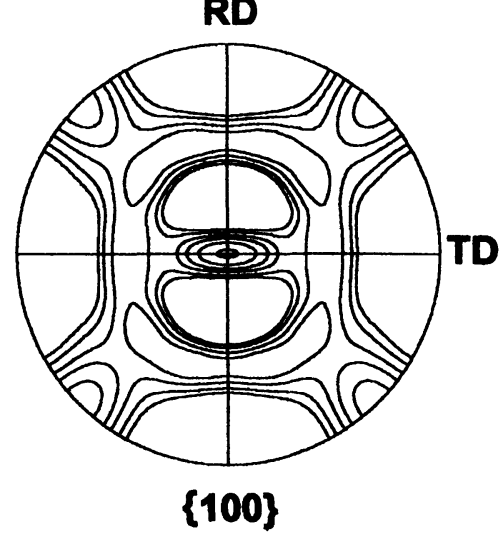

Figure 5 The simulated texture obtained by the self consistent model at $50 \%$ thickness reduction. Hardening is accounted for and initial values of the reference shear stress were: $\tau_{0}^{110}=192 \mathrm{MPa}$, $\tau_{0}^{112}=187 \mathrm{MPa}, \tau_{0}^{332}=527 \mathrm{MPa}$. Isovalues in the ODF: $0.7,1.0,1.4,2.0,2.8,4.0,5.6,8.0$. Isovalues in the pole figures: $0.8,1.0,1.3,1.6,2.0,2.5$. 
evident that all the important features of the experimental texture are reproduced (if the ghost components produced by the $\omega$ phase are neglected near to the $\phi=90^{\circ}$ plane). The two fibres (RD and ND) are well developed and two ideal orientations along the RD fibre $\{(001)$ [110] and (111) [110]\} are particularly strong. The relative activity of the different slip system families was also calculated (taken to be proportional to the accummulated slips) and the following results have been obtained: $54 \%$ for the $\{110\}$ slip systems, $45 \%$ for the $\{112\}$ slip systems, and $1 \%$ for the $\{332\}$ twinning systems. These values remain nearly constant during deformation.

\subsection{The Effect of Hardening}

In order to examine the influence of the microscopic hardening on the development of the texture, hardening has been eliminated by keeping the initial critical stresses constant during deformation. The results obtained in this way are shown in Figure 6. One can see that the (111) [110] component has become too strong with respect to the experience (compare to Figure 3). Nevertheless, the main features of the texture are more or less reproduced. Therefore, hardening is not a decisive factor in the texture development but is necessary to achieve a better accuracy.
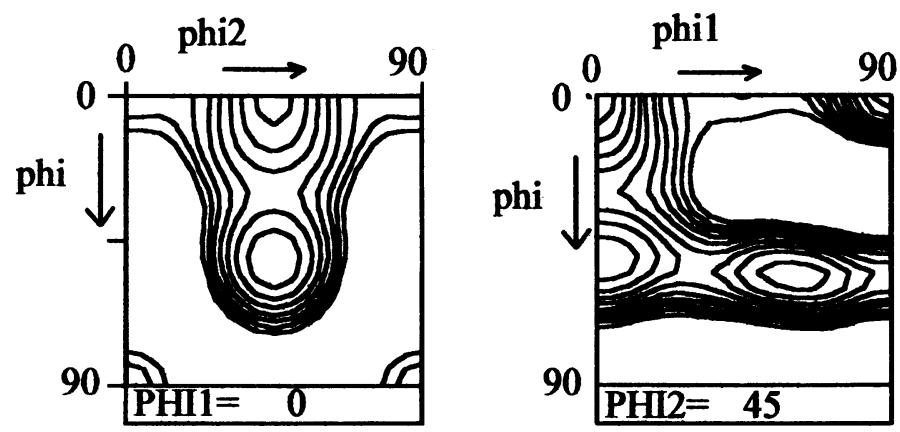

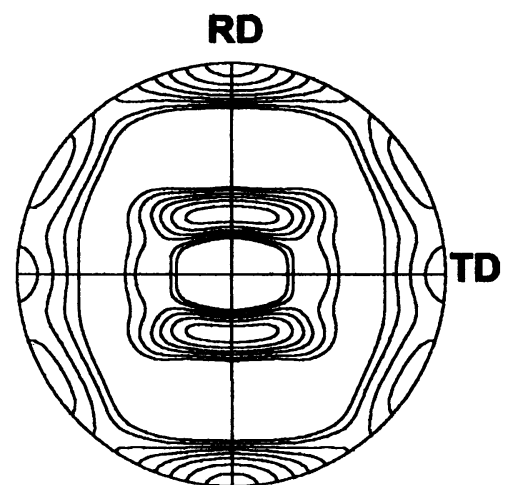

$\{110\}$

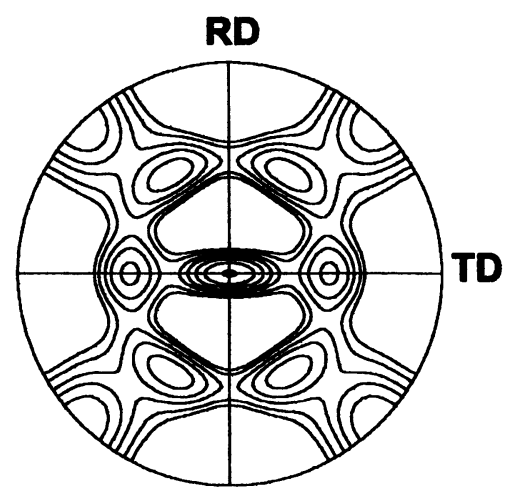

$\{100\}$

Figure 6 Predicted texture by the self consistent model without hardening (i.e. by using constant reference stresses, given in Figure 5). Isovalues in the ODF: 0.7, 1.0, 1.4, 2.0, 2.8, 4.0, 5.6, 8.0, 11.0. Isovalues in the pole figures: $0.8,1.0,1.3,1.6,2.0,2.5,3.2,4$. 

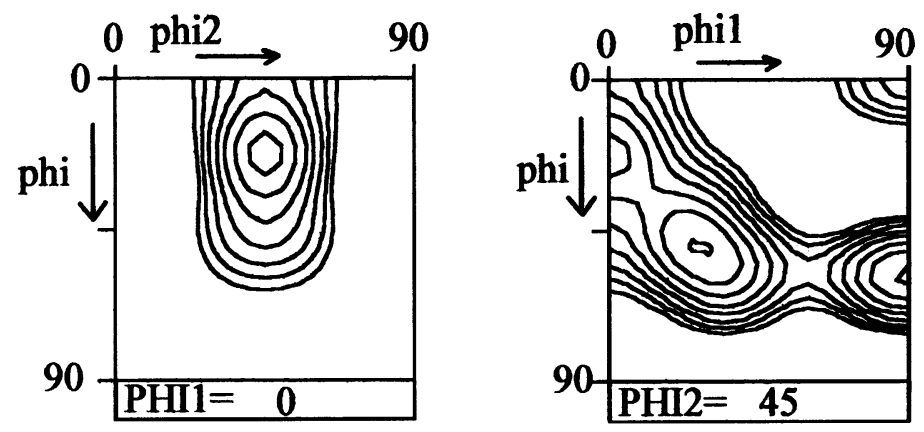

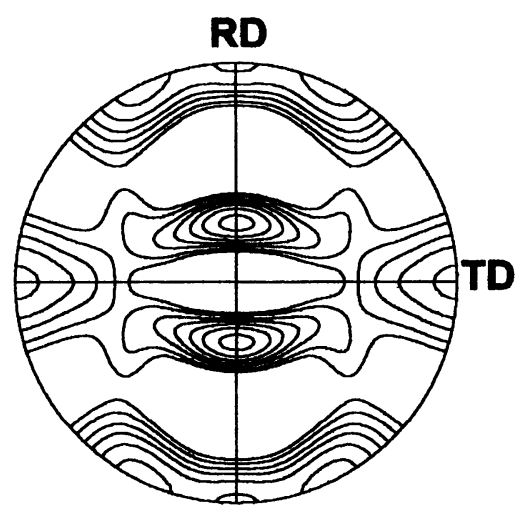

$\{110\}$

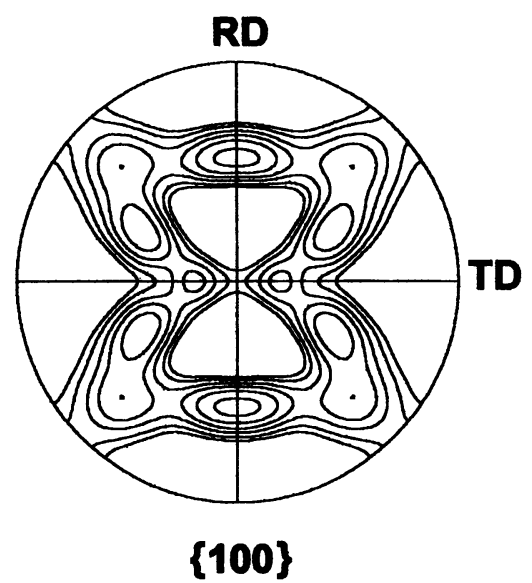

Figure 7 Texture predicted by the self consistent model with only the $\{110\}$ slip and $\{332\}$ twinning systems $(\{112\}$ excluded). Isovalues in the ODF: $0.7,1.0,1.4,2.0,2.8,4.0,5.6,8.0$. Isovalues in the pole figures: $0.8,1.0,1.3,1.6,2.0,2.5,3.2$.

\subsection{The Effect of $\{112\}<111>$ Slip on Texture Development}

When the $\{112\}<111>$ slip system family is eliminated, the obtained texture is very far from the experimental one, see Figure 7. Thus, the 24 other deformations systems alone are not able to predict a correct texture development. Several simulations have been carried out by varying the reference stresses of the slip systems. It has been found that if the initial critical stresses of the two families of slip systems are taken quite different, it is no longer possible to approach well the experimental texture development. The best results were obtained (Figure 5) when the critical stresses given in Section 3.3. were used, which represents a relative strengths of 0.97 of the $\{112\}$ systems with respect to the $\{110\}$ family. Similar relative value of the critical stresses has also been found in steel (Daniel and Jonas (1990)).

\subsection{Influence of the $\{332\}<113>$ Twinning Activity on the Texture Development}

According to the experimental observations, the present alloy shows high activity of twinning. However, in the simulation presented in section 4.2 . above, only $1 \%$ volume fraction of the grains were transformed into their twin orientations. For the purpose 

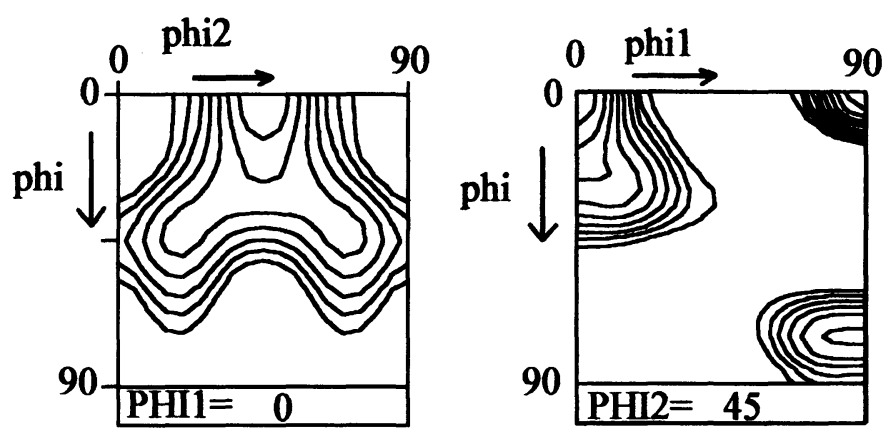

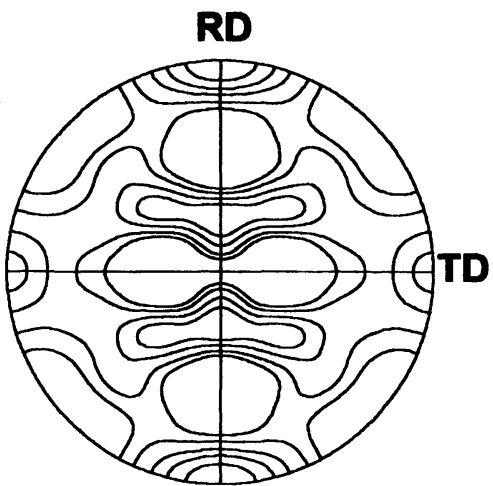

$\{110\}$

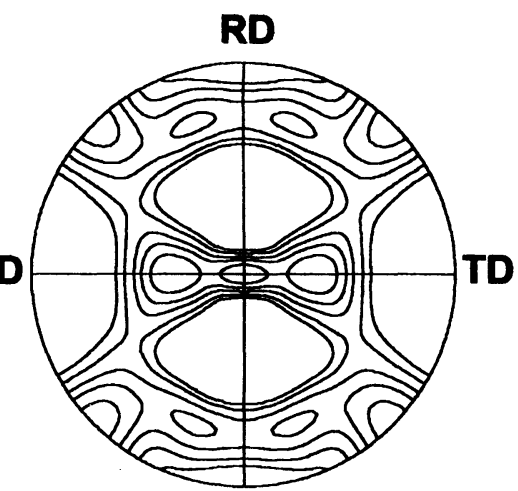

$\{100\}$

Figure 8 Texture predicted by the self consistent model with enhanced activity of twinning (up to $40 \%$ volume fraction). Isovalues in the ODF: $1.0,1.3,2.0,2.5,3.2,4.0,5.0,6.4,8$. Isovalues in the pole figures: $0.8,1.0,1.3,1.6,2.0,2.5$.

of increasing the activity of twinning, the initial reference stresses of the $\{33 \overline{2}\}$ $<113>$ twinning family was decreased, without changing the initial reference stresses of the other systems: the value $\tau_{0}^{332}=200 \mathrm{MPa}$ was adopted. With this critical stress, the volume fraction of twinning has increased up to $40 \%$. This value seems to be in better agreement with the results of the metallographic observations. However, the deformation texture is not reproduced. As can be seen from Figure 8, the ND fibre does not exist. Other tests carried out with $\tau_{0}^{332}$ varying in the range between $200 \mathrm{MPa}$ and $400 \mathrm{MPa}$ have never led to good agreements with the measured texture as soon as the twinning volume fraction was significant.

\subsection{Simulation of Twinning in Two Steps}

The measured twinning activity as a function of the deformation is shown in Figure 9 (Nicotra and Philippe (1994a)). As a measure of the twinning activity in the experiment, the surface fraction of twinned grains with respect to all the examined grains was calculated. One can identify two stages in this figure. At the beginning, up to $20 \%$ strain, the activity is nearly linear and then increases only slightly at larger strains. However, the results of all simulations showed an evenly increasing volume fraction 


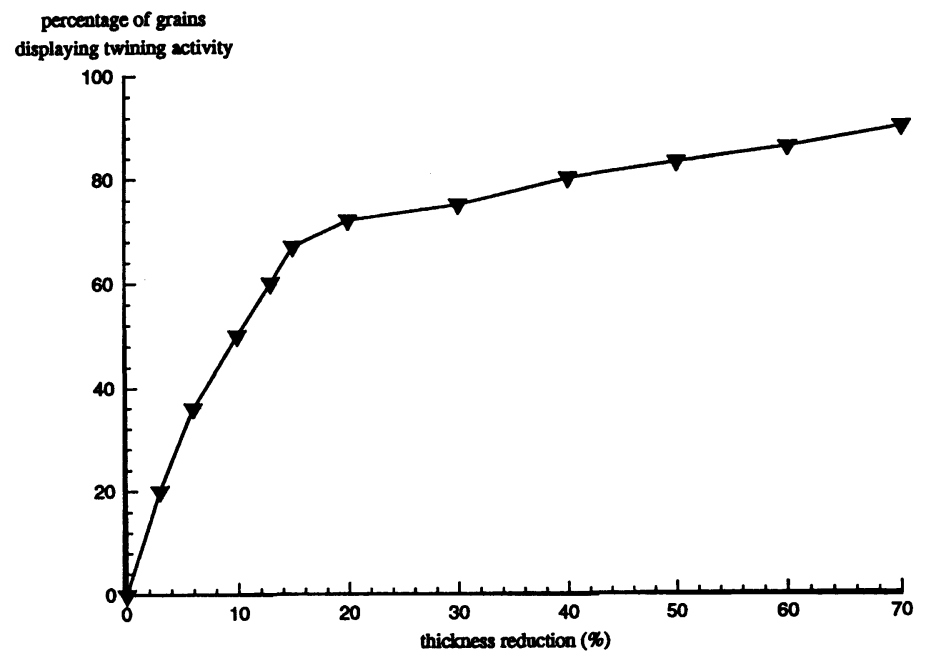

Figure 9 Measured twinning activity as a function of thickness reduction.

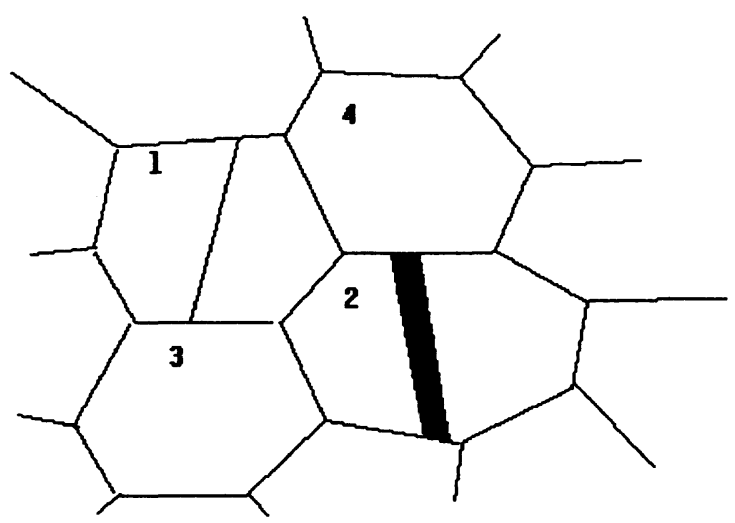

Figure 10 Definition of the deformation twinning volume fraction, $f_{t w}$. In grain $1: f_{t w}=0$, in grain 2: $f_{t w}=$ black area/grain surface.

for twinning. That is, when the critical stresses are fixed at the beginning of the deformation, the corresponding activities of the deformation modes stay nearly constant. The metallographic observations carried out on the rolled sample have concluded that $80 \%$ of the grains have developed at least one twin. According to the author's opinion, this percentage does not describe correctly the way twins modify the relative volume fractions in the texture. When deformation twins are formed, the twinned volume is bordered by straight planes (Figure 10) and only that part of the grain is transformed into its twin position which is situated between these limiting planes (which are in fact new grain boundaries). The twinned volume fraction therefore has to be calculated as the ratio of the total surface of the twins and that of the grain (if the form of the grain is assumed to be cubic). In this way, the twinned volume fraction was estimated to be about $20 \%$ at a rolling reduction of $10 \%$. 


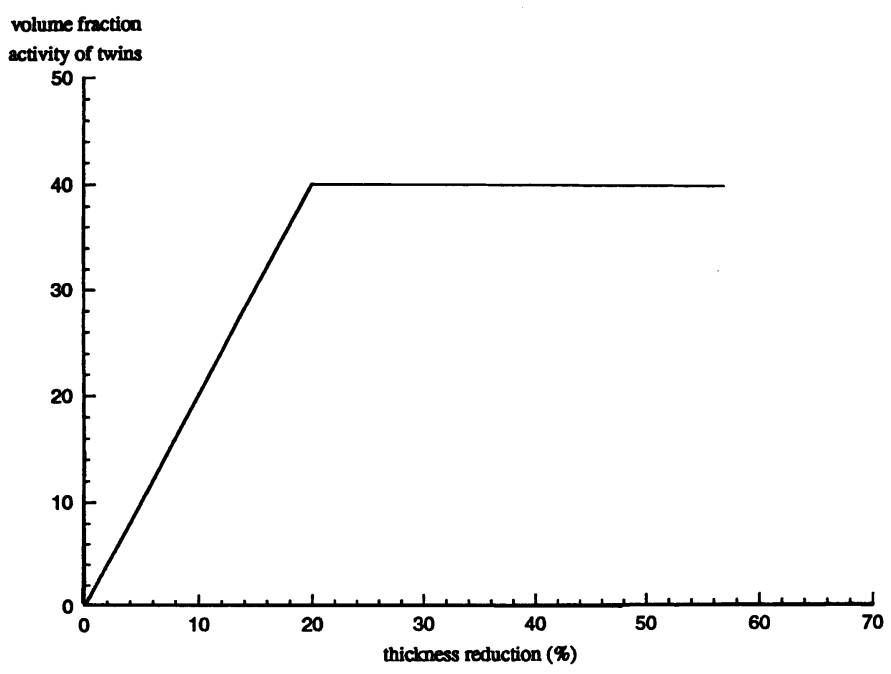

Figure 11 Approximation of the experimental twinning activity by two stages.

The experimental twinning activity shows two stages, see Figure 9. This observation will be idealized now for simulation purposes and the volume fraction variation of twins is approximated by the curve shown Figure 11. It means that the twinning activity is assumed to increase up to $40 \%$ at a strain of $20 \%$ and then it is stopped.

To model this two stage twinning activity, the simulation was done in two steps. In the first part, the initial reference stress of the twin family was taken so that the twinned volume fraction was equal to $40 \%$ at 0,2 strain. The initial critical stresses of the other two slip families were not modified. In the second part of the calculation, the $\tau_{0}^{332}$ value was changed to a large value $(1000 \mathrm{MPa})$ in every grain and then the calculation was completed up to $\varepsilon=0.8$. The results obtained in this way are shown in Figure 12. It can be seen that they are in good agreement with the experimental texture. In comparison with the ODF obtained in section 4.2. (Figure 5), a significant improvement can be noticed: the (001) [1히 orientation is less pronounced and the (111) $[1 \overline{1} 0]$ component is strengthened.

\subsection{Comparison with a Taylor Type Calculation}

Simulations were also performed using the Taylor model, employing the same macroscopic parameters as in the self consistent case. The results obtained are shown in Figure 13. As can be seen, the texture is no longer predicted satisfactorily with a Taylor scheme. The $<111>$ fibre appears in a shifted position and the ideal orientations are also not reproduced. It is clear that the deformation texture as predicted by the self consistent model is in much better agreement with the experiment as the one obtained by the Taylor model. 


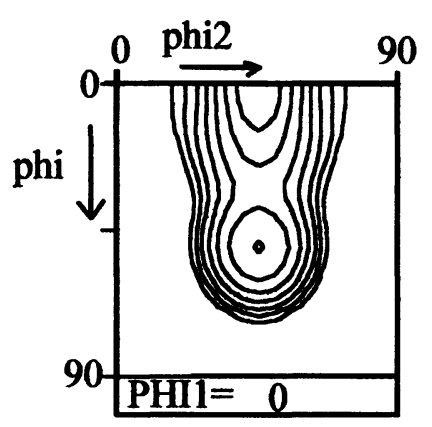

RD

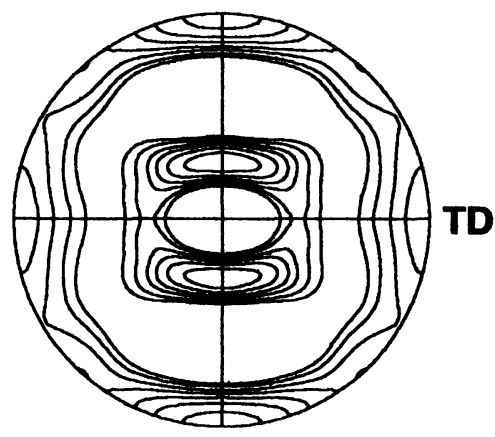

$\{110\}$
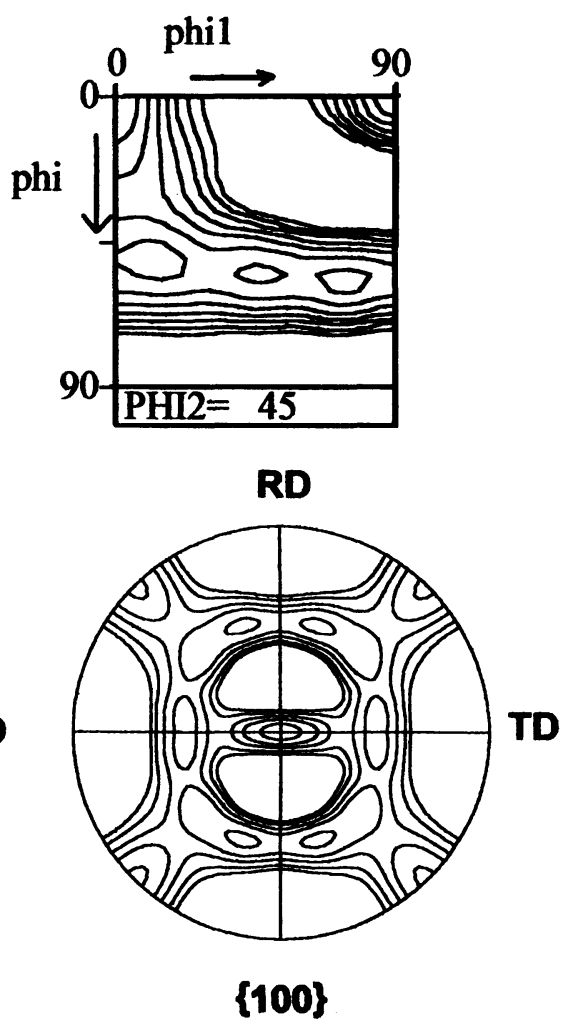

Figure 12 Predicted texture at $50 \%$ thickness reduction predicted by the self consistent model, carried out in two steps. In the first step (up to a $20 \%$ logarithmic strain) the twinning activity was kept at $40 \%$ volumic fraction and then twinning was eliminated. Isovalues in the ODF: $0.7,1.0,1.4,2.0$, 2.8, 4.0, 5.6. Isovalues in the pole figure: $0.8,1.0,1.3,1.6,2.0,2.5,3.2$.

\section{CONCLUSIONS}

In the present work, an application of the self consistent polycrystal approach proposed by Molinari and Tóth (1994) has been carried out for the modelisation of the texture development in a Ti20V alloy during rolling. First the characteristics of the model have been discussed and then the model parameters were identified using the stress-strain curves obtained in tension. The $\{110\}$ and $\{112\}$ slip systems were employed together with the $\{332\}$ twinning family. The results obtained by the simulations in rolling were compared to experiments, from which comparison the following main conclusions can be drawn:

1. The experimental texture development can be well reproduced by self consistent model with an initial strengths of the $\{110\},\{112\}$ slip and $\{332\}$ twinning systems of $192 \mathrm{MPa}, 187 \mathrm{MPa}$ and $527 \mathrm{MPa}$, respectively. Any significant deviation from the relative strengths of the different deformation mechanisms leads to significant differences as compared to the experimental texture development. 


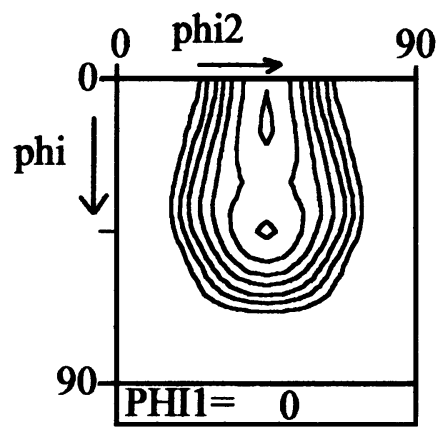

RD

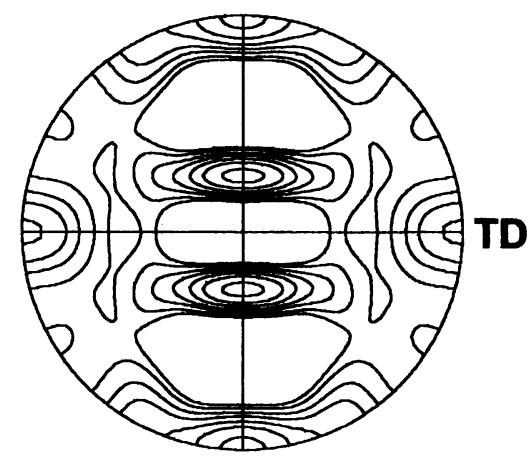

\{110\}
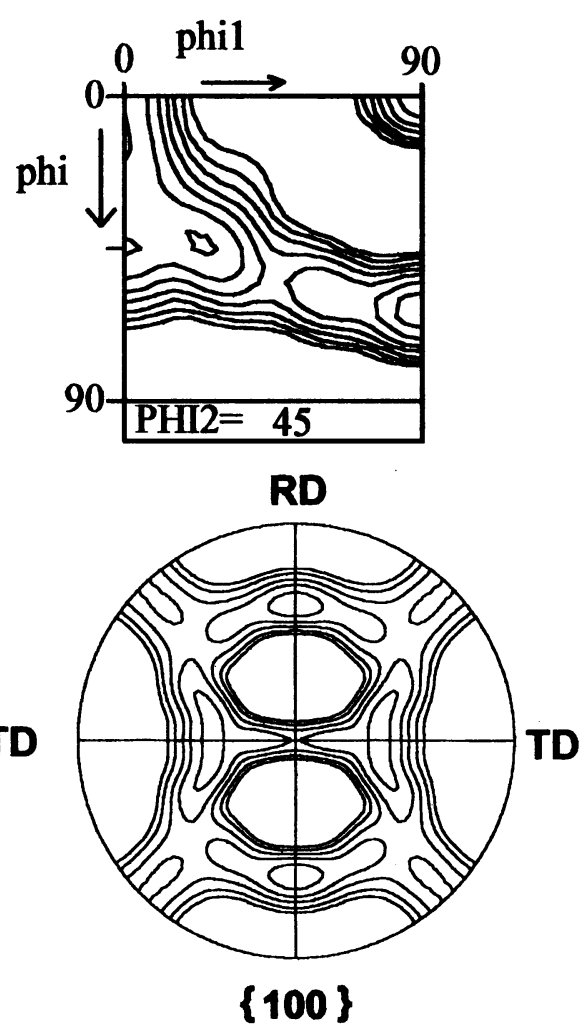

Figure 13 Texture at $50 \%$ thickness reduction by the viscoplastic Taylor model. Isovalues in the ODF: $0.7,1.0,1.4,2.0,2.8,4.0,5.6$. Isovalues in the pole figures: $0.8,1.0,1.3,1.6,2.0,2.5,3.2$.

2. The activity of twinning produces only about $1 \%$ volume fraction - transfer of the grains into their twin orientations when the twinning systems are considered to harden in the same way as the slip systems. Some improvement in the texture evolution can be verified when twinning is enhanced at the beginning of deformation ( $40 \%$ volume fraction up to $20 \%$ thickness reduction) and then supressed at larger strains.

\section{Acknowledgements}

The authors are grateful to Dr. A. Nicotra and Prof. M. J. Philippe for providing the experimental information on the Ti-20V material and for the stimulating discussions on the role of the slip and twinning deformation mechanisms.

\section{References}

Bronkhorst, C. A., Kalidindi, S. R. and Anand, L. (1992). Crystallographic Texture Evolution in Bulk Deformation Processing of FCC Metals, Phil. Trans. Roy. Soc. London, A341, 443.

Daniel, D. and Jonas, J. J. (1990). Measurement and Prediction of Plastic Anisotropy in Deep Drawing Steels, Met. Trans., 21A, 331-343. 
Eshelby, J. D. (1957). The determination of the elastic field of an ellipsoidal inclusion, and related problems, Procs. Roy. Soc. London, A241, 376-396.

Guibert, J. P. (1994). personal communication, ETCA, Arcueil, France.

Hanada, S., Ozeki, M. and Izumi, O. (1985). Deformation Characteristics in $\beta$ Phase Ti-Nb Alloys, Met. Trans., 16A, 789-795.

Mathur, K. K. and Dawson, P. R. (1989). On Modeling the Development of Crystallographic Texture in Bulk Deformation Processes, Int. J. Plasticity, 5, 67-94.

Molinari, A., Canova, G. and Ahzi, S. (1987). A Self Consistent Approach of the Large Deformation Polycrystal Plasticity, Acta Metall. Mater., 35, 2983-2994.

Molinari, A. and Tóth, L. S. (1994). Tuning a Self Consistent Viscoplastic Model by Finite Element Results, Part I: Modeling Acta Metall. Mater., 42, 2453-2458.

Nicotra, A. and Philippe, M. J. (1994a). Experimental studies on the Ti20V alloy, personal communication, Université de Metz, France.

Nicotra, A. and Philippe, M. J. (1994b). Strain Mechanisms and Mechanical Properties of Various $\beta$ Phase Titanium Alloys, In Beta Titanium Alloys, Editions de la revue Metallurgie, vol. 8, Société Française de Métallurgie et de Matériaux, Eds. A. Vassel, D. Eylon and Y. Combres, pp. 189-196.

Richman, R. H. (1964). The Diversity of Twinning in Body-Centered Cubic Structures, In Deformation Twinning, Metallurgical Society Conferences, Gordon and Breach Science Publishers, New York, London, vol. 25, pp. 237-271.

Tomé, C. N., Lebensohn, R. A. and Kocks, U. F. (1991). A Model for Texture Development Dominated by Deformation Twinning: Application to Zirconium Alloys, Acta Metall. Mater, 39, 2667.

Tóth, L. S., Jonas, J. J., Daniel, D. and Ray, R. K. (1990). Development of Ferrite Rolling Textures in Low- and Extra Low-Carbon Steels, Met. Trans., 21A, 2985-3000.

Tóth, L. S. and Van Houtte, P. (1992). Dicretization Textures for Orientation Distribution Functions, Textures and Microstructures, 19, 229-244.

Tóth, L. S. and Molinari, A. (1994). Tuning a Self Consistent Viscoplastic Model by Finite Element Results, Part II: Application to Torsion Textures, Acta Metall. Mater., 42, 2459-2466.

Van Houtte, P. (1978). Simulation of the Rolling and Shear Texture of Brass by the Taylor Theory Adapted for Mechanical Twinning, Acta Metall., 26, 591-604.

Van Houtte, P. ODF Software, Katholieke Universiteit Leuven, Belgium.

Zhou, Y., Neale, K. W., Tóth, L. S. (1993). A Modified Model of Latent Hardening for Rate-Dependent FCC Polycrystals, Int. J. Plasticity, 9, 961-978. 